\title{
Causes and Management of Insect and Mite Resistance in Strawberry Production ${ }^{1}$
}

\author{
James F. Price and Curtis Nagle ${ }^{2}$
}

Resistance of arthropods to crop management chemicals has been problematic since the early era of synthetic organic pesticides. During the late 1990s, the twospotted spider mite (Tetranychus urticae Koch) became resistant to abamectin, the miticide used in strawberry culture. Since then, several new miticides, including acequinocyl, bifenazate, etoxazole, fenpyroximate, hexythiazox, and spiromesifen, have been integrated into strawberry production; overuse of abamectin has ceased; and abamectin once again is effective in rotation with the new materials. This latter development could prove temporary, especially if growers again use abamectin regularly.

Poor performance of pesticides does not always indicate pest resistance. Such factors as pesticide degradation in storage, hydrolysis in acid or alkaline preparations, applications to an incorrect life stage of the pest, or other inadequate application procedures may contribute to poor control.

\section{A Definition of Resistance}

Pest populations can be susceptible or resistant to a pesticide. Resistance occurs when a formerly susceptible pest population becomes significantly less susceptible to a properly applied pesticide. Pesticide resistance is a population-based phenomenon in which the group genetic composition shifts and individuals with genes that confer resistance to a pesticide begin to dominate the population, reducing the pesticide's effectiveness .

\section{Establishment of Resistance}

Resistant populations are protected from formerly effective pesticides through one or more means. For example, resistant pests may: (1) deactivate (break down) the toxin; (2) sequester the toxin (safely store it within their bodies); (3) avoid the toxin; (4) excrete the toxin from their bodies more effectively; (5) have an altered target site that will not accumulate the toxin; or (6) reduce the permeability by the toxin through their exoskeletons ("shells").

Individuals within a susceptible pest population often vary in their level of susceptibility; however, the non-susceptible type occurs only very rarely. When a pesticide is applied repeatedly, the susceptible pests die and the resistant ones survive, mate with other survivors and reproduce. Some of their offspring inherit the parents' characteristic for survival. Upon additional applications, the susceptible offspring within the remaining population die and the resistant ones survive, mate with other survivors and produce more offspring. Further applications additionally select for the resistant individuals until that form (genotype) is common. The population then is regarded as resistant and the effectiveness of the pesticide is lost.

\section{Resistance Management}

Resistance can develop rapidly with pests that have many generations per year, when multiple generations are exposed to a pesticide, and when new individuals do

1. This document is ENY-841 (IN713), a publication of the Department of Entomology and Nematology, Florida Cooperative Extension Service, IFAS, University of Florida. Publication date November 2007. Revised November 2012. Please visit the EDIS website at http://edis.ifas.ufl.edu.

2. James F. Price, associate professor, Gulf Coast Research and Education Center; and Curtis Nagle, biological scientist, Gulf Coast Research and Education Center. Cooperative Extension Service, IFAS, University of Florida, Gainesville, FL 32611. 
not move into a treated area to dilute the frequency of the resistant genes. Some of these factors occurred on strawberry farms in the 1990s, contributing to development of abamectin resistance in spider mites.

The main objectives of on-farm resistance management programs should be to minimize the number of exposures of pests to pesticides with a similar mode of action and to use non-chemical approaches to arthropod management. (Mode of action is the specific activity of the toxin that results in the death of the pest. For instance, one mode of action is to inhibit mitochondrial complex I electron transport. This kills pests by preventing them from producing energy in affected cells, essentially "starving" them.)

Repeated exposures to a pesticide are the primary drivers of resistance, but much can be done to manage pests by means other than chemicals. Care can be taken to rotate strawberries with other crops, use pest-resistant varieties, plant pest-free transplants, conserve and release natural enemies, etc. Pest-specific tactics are available for particular situations; for instance, removal of all ripe strawberries from a field will eliminate reproductive sites for sap beetles.

Strawberry fields should be scouted weekly and pesticide applications made only when pest densities approach economic injury levels. When pesticide use is required, products should be rotated among the different modes of action indicated on many modern product labels. A list of modes of action can be found by selecting "MoA Classification Scheme" at the Insecticide Resistance Action Committee Website: http://www.irac-online.org [version 7.2 April, 2012].

Tables 1through 3 present insecticide and miticide modes of action summaries for Florida strawberry production. Sound rotation plans often recommend pesticides of one mode of action for one pest generation and a pesticide of a different mode of action for another generation. If multiple pesticide applications are required, rotations should continue through all practical modes of action before returning to a previously used one. The use of certain unique products with known general modes of action (such as soaps and oils) is unlikely to result in pest resistance, and no codes are assigned these products. These products can be used without regard to a rotation plan for resistance management.
When pesticides are used, it is important to assure that fresh, fully potent pesticides are prepared and applied in accordance with label directions. Aqueous pesticidal preparations should be adjusted to near neutral $\mathrm{pH}(\mathrm{pH}$ 7.0) or as specified by the label. Sprayer calibration, nozzle condition and pressure, and spray placement must be correct. Applications also should be timed and directed to contact the most susceptible life stage of the pest.

\section{Conclusion}

Episodes of pest resistance to popular pesticides can cause yield losses, reduction of fruit quality, added control costs, environmental degradation, and emotional stress among farmers. These consequences can be alleviated if resistance management is practiced throughout the strawberry industry. If they minimize pesticide application by depending more on biological and cultural pest control measures, and take care not to expose pest populations to pesticides with identical modes of action, growers can avoid causing pesticide resistance. 
Table 1. Mode of action of insecticides and miticides registered for use in Florida's strawberry crops (presented by active ingredient). (Insecticide Resistance Action Committee (IRAC) mode of action classification codes version 7.2).

\begin{tabular}{|c|c|c|}
\hline Active Ingredient (common name) & Trade Name Examples & Mode of Action Code \\
\hline \multirow[t]{2}{*}{ 1,3-dichloropropene } & Inline & \multirow[t]{2}{*}{ no code ${ }^{a}$} \\
\hline & Telone & \\
\hline \multirow[t]{2}{*}{ abamectin } & Abacus & \multirow[t]{2}{*}{6} \\
\hline & Agri-Mek & \\
\hline acequinocyl & Kanemite & $20 \mathrm{~B}$ \\
\hline acetamiprid & Assail & $4 \mathrm{~A}$ \\
\hline avermectin \& bifenthrin & Athena & $6 \& 3 A$ \\
\hline \multirow[t]{2}{*}{ azadirachtin } & Aza-Direct & \multirow[t]{2}{*}{$u^{b}$} \\
\hline & Azatrol & \\
\hline \multirow[t]{2}{*}{ Bacillus thuringiensis aizawai } & Agree & \multirow[t]{2}{*}{$11 \mathrm{~A}$} \\
\hline & Xentari & \\
\hline \multirow[t]{2}{*}{ Bacillus thuringiensis kurstaki } & Dipel & \multirow[t]{2}{*}{$11 \mathrm{~A}$} \\
\hline & Javelin & \\
\hline \multirow[t]{3}{*}{ Beauveria bassiana } & Botanigard & \multirow[t]{3}{*}{ no code } \\
\hline & Mycotrol & \\
\hline & Naturalis & \\
\hline bifenazate & Acramite & un \\
\hline bifenthrin & Brigade & $3 \mathrm{~A}$ \\
\hline bifenthrin \& avermectin & Athena & $3 A \& 6$ \\
\hline bifenthrin \& imidacloprid & Brigadier & $3 \mathrm{~A} \& 4 \mathrm{~A}$ \\
\hline buprofezin & Courier & 16 \\
\hline carbaryl & Sevin & $1 \mathrm{~A}$ \\
\hline chlorantraniliprole & Coragen & 28 \\
\hline chlorantraniliprole \& thiamethoxam & Voliam Flexi & $28 \& 4 A$ \\
\hline \multirow[t]{2}{*}{ chlorpyrifos } & Govern & \multirow[t]{2}{*}{ 1B } \\
\hline & Lorsban & \\
\hline Chromobacterium subtsugae & MBI-203 & no code \\
\hline clarified hydrophobic extract of neem oil & Trilogy & no code \\
\hline diazinon & Diazinon & 1B \\
\hline etoxazole & Zeal & $10 \mathrm{~B}$ \\
\hline fenbutatin oxide & Vendex & $12 \mathrm{~B}$ \\
\hline fenpropathrin & Danitol & $3 \mathrm{~A}$ \\
\hline fenpyroximate & Portal & $21 \mathrm{~A}$ \\
\hline flubendamide & Synapse & 28 \\
\hline hexythiazox & Savey & $10 \mathrm{~A}$ \\
\hline \multirow[t]{2}{*}{ imidacloprid } & Admire & \multirow[t]{2}{*}{$4 \mathrm{~A}$} \\
\hline & Provado & \\
\hline imidacloprid \& bifenthrin & Brigadier & $4 A \& 3 A$ \\
\hline iron phosphate & Sluggo & no code \\
\hline \multirow[t]{2}{*}{ Isaria fumosorosea } & PFR-97 & \multirow[t]{2}{*}{ no code } \\
\hline & Preferal & \\
\hline malathion & Malathion & $1 \mathrm{~B}$ \\
\hline \multirow[t]{2}{*}{ metaldehyde } & Deadline & \multirow[t]{2}{*}{ no code } \\
\hline & Slug-Fest & \\
\hline
\end{tabular}




\begin{tabular}{|c|c|c|}
\hline Active Ingredient (common name) & Trade Name Examples & Mode of Action Code \\
\hline metam sodium & Vapam & no code \\
\hline methoxyfenozide & Intrepid & 18 \\
\hline naled & Dibrom & $1 \mathrm{~B}$ \\
\hline novaluron & Rimon & 15 \\
\hline \multirow[t]{2}{*}{ potassium salts of fatty acids } & AllPro Insecticidal Soap & \multirow[t]{2}{*}{ no code } \\
\hline & M-Pede & \\
\hline \multirow[t]{4}{*}{ pyrethrins } & Diatect & \multirow[t]{4}{*}{$3 \mathrm{~A}$} \\
\hline & PyGanic & \\
\hline & Pyrenone & \\
\hline & Pyreth-It & \\
\hline pyrethrins \& rotenone & Pyrellin & $3 A \& 21 B$ \\
\hline pyriproxyfen & Esteem & $7 C$ \\
\hline refined petroleum distillate & Ultra-Fine Oil & no code \\
\hline rotenone \& pyrethrins & Pyrellin & $21 B \& 3 A$ \\
\hline s-methoprene & Extinguish & $7 \mathrm{~A}$ \\
\hline sorbitol octanoate & SorbiShield & no code \\
\hline spinetoram & Radiant & 5 \\
\hline \multirow[t]{2}{*}{ spinosad } & Entrust & \multirow[t]{2}{*}{5} \\
\hline & Spintor & \\
\hline spiromesifen & Oberon & 23 \\
\hline sucrose octanoate & SucraShield & no code \\
\hline \multirow[t]{2}{*}{ thiamethoxam } & Actara & \multirow[t]{2}{*}{$4 \mathrm{~A}$} \\
\hline & Platinum & \\
\hline thiamethoxam \& chlorantraniliprole & Voliam Flexi & $4 A \& 28$ \\
\hline
\end{tabular}


Table 2. Mode of action of insecticides and miticides registered for use in Florida's strawberry crops (presented by mode of action code). (Insecticide Resistance Action Committee mode of action classification code version 7.2).

\begin{tabular}{|c|c|c|}
\hline Mode of Action Code & Active Ingredient (common name) & Trade Name Examples \\
\hline \multirow[t]{18}{*}{ no code ${ }^{a}$} & \multirow[t]{2}{*}{ 1,3-dichloropropene } & Inline \\
\hline & & Telone \\
\hline & \multirow[t]{3}{*}{ Beauveria bassiana } & Botanigard \\
\hline & & Mycotrol \\
\hline & & Naturalis \\
\hline & Chromobacterium subtsugae & MBI-203 \\
\hline & clarified hydrophobic extract of neem oil & Trilogy \\
\hline & iron phosphate & Sluggo \\
\hline & \multirow[t]{2}{*}{ Isaria fumosorosea } & PFR-97 \\
\hline & & Preferal \\
\hline & \multirow[t]{2}{*}{ metaldehyde } & Deadline \\
\hline & & Slug-Fest \\
\hline & metam sodium & Vapam \\
\hline & \multirow[t]{2}{*}{ potassium salts of fatty acids } & AllPro Insecticidal Soap \\
\hline & & M-Pede \\
\hline & refined petroleum distillate & Ultra-fine Oil \\
\hline & sorbitol octanoate & SorbiShield \\
\hline & sucrose octanoate & SucraShield \\
\hline $1 \mathrm{~A}$ & carbaryl & Sevin \\
\hline \multirow[t]{5}{*}{ 1B } & \multirow[t]{2}{*}{ chlorpyrifos } & Govern \\
\hline & & Lorsban \\
\hline & diazinon & Diazinon \\
\hline & malathion & Malathion \\
\hline & naled & Dibrom \\
\hline \multirow[t]{6}{*}{$3 \mathrm{~A}$} & bifenthrin & Brigade \\
\hline & fenpropathrin & Danitol \\
\hline & \multirow[t]{4}{*}{ pyrethrins } & Diatect \\
\hline & & PyGanic \\
\hline & & Pyrenone \\
\hline & & Pyreth-It \\
\hline $3 A \& 21 B$ & pyrethrins \& rotenone & Pyrellin \\
\hline $3 A \& 4 A$ & bifenthrin \& imidacloprid & Brigadier \\
\hline $3 A \& 6$ & bifenthrin \& avermectin & Athena \\
\hline \multirow[t]{5}{*}{$4 \mathrm{~A}$} & acetamiprid & Assail \\
\hline & \multirow[t]{2}{*}{ imidacloprid } & Admire \\
\hline & & Provado \\
\hline & \multirow[t]{2}{*}{ thiamethoxam } & Actara \\
\hline & & Platinum \\
\hline $4 A \& 28$ & thiamethoxam \& chlorantraniliprole & Voliam Flexi \\
\hline $4 A \& 3 A$ & imidacloprid \& bifenthrin & Brigadier \\
\hline \multirow[t]{3}{*}{5} & spinetoram & Radiant \\
\hline & \multirow[t]{2}{*}{ spinosad } & Entrust \\
\hline & & Spintor \\
\hline
\end{tabular}




\begin{tabular}{|c|c|c|}
\hline Mode of Action Code & Active Ingredient (common name) & Trade Name Examples \\
\hline \multirow[t]{2}{*}{6} & \multirow[t]{2}{*}{ abamectin } & Abacus \\
\hline & & Agri-Mek \\
\hline $6 \& 3 A$ & avermectin \& bifenthrin & Athena \\
\hline $7 \mathrm{~A}$ & s-methoprene & Extinguish \\
\hline $7 C$ & pyriproxyfen & Esteem \\
\hline $10 \mathrm{~A}$ & hexythiazox & Savey \\
\hline $10 \mathrm{~B}$ & etoxazole & Zeal \\
\hline \multirow[t]{4}{*}{$11 \mathrm{~A}$} & \multirow[t]{2}{*}{ Bacillus thuringiensis aizawai } & Agree \\
\hline & & Xentari \\
\hline & \multirow[t]{2}{*}{ Bacillus thuringiensis kurstaki } & Dipel \\
\hline & & Javelin \\
\hline $12 B$ & fenbutatin oxide & Vendex \\
\hline 15 & novaluron & Rimon \\
\hline 16 & buprofezin & Courier \\
\hline 18 & methoxyfenozide & Intrepid \\
\hline $20 B$ & acequinocyl & Kanemite \\
\hline $21 \mathrm{~A}$ & fenpyroximate & Portal \\
\hline $21 B \& 3 A$ & rotenone \& pyrethrins & Pyrellin \\
\hline 23 & spiromesifen & Oberon \\
\hline \multirow[t]{2}{*}{28} & chlorantraniliprole & Coragen \\
\hline & flubendamide & Synapse \\
\hline $28 \& 4 \mathrm{~A}$ & chlorantraniliprole \& thiamethoxam & Voliam Flexi \\
\hline \multirow[t]{3}{*}{$u n^{b}$} & \multirow[t]{2}{*}{ azadirachtin } & Aza-Direct \\
\hline & & Azatrol \\
\hline & bifenazate & Acramite \\
\hline
\end{tabular}


Table 3. Mode of action of insecticides and miticides registered for use in Florida's strawberry crops (presented by trade name). (Insecticide Resistance Action Committee mode of action classification codes version 7.2).

\begin{tabular}{|c|c|c|}
\hline Trade Name Examples & Active Ingredient (common name) & Mode of Action Code \\
\hline Abacus & abamectin & 6 \\
\hline Acramite & bifenazate & $u^{a}$ \\
\hline Actara & acetamiprid & $4 \mathrm{~A}$ \\
\hline Admire & imidacloprid & $4 \mathrm{~A}$ \\
\hline Agree & Bacillus thuringiensis aizawai & $11 \mathrm{~A}$ \\
\hline Agri-Mek & abamectin & 6 \\
\hline AllPro Insecticidal Soap & potassium salts of fatty acids & no code ${ }^{b}$ \\
\hline Assail & acetamiprid & $4 \mathrm{~A}$ \\
\hline Athena & avermectin \& bifenthrin & $6 \& 3 A$ \\
\hline Aza-Direct & azadirachtin & un \\
\hline Azatrol & azadirachtin & un \\
\hline Botanigard & Beauveria bassiana & no code \\
\hline Brigade & bifenthrin & $3 \mathrm{~A}$ \\
\hline Brigadier & bifenthrin \& imidacloprid & $3 A \& 4 A$ \\
\hline Coragen & chlorantraniliprole & 28 \\
\hline Courier & buprofezin & 16 \\
\hline Danitol & fenpropathrin & $3 \mathrm{~A}$ \\
\hline Deadline & metaldehyde & no code \\
\hline Diatect & pyrethrins & $3 A$ \\
\hline Dipel & Bacillus thuringiensis kurstaki & $11 \mathrm{~A}$ \\
\hline Diazinon & diazinon & $1 \mathrm{~B}$ \\
\hline Dibrom & naled & $1 \mathrm{~B}$ \\
\hline Entrust & spinosad & 5 \\
\hline Esteem & pyriproxyfen & $7 C$ \\
\hline Extinguish & s-methoprene & $7 \mathrm{~A}$ \\
\hline Govern & chlorpyrifos & $1 \mathrm{~B}$ \\
\hline Intrepid & methoxyfenozide & 18 \\
\hline InLine & 1,3-dichloropropene & no code \\
\hline Javelin & Bacillus thuringiensis kurstaki & $11 \mathrm{~A}$ \\
\hline Kanemite & acequinocyl & $20 B$ \\
\hline Lorsban & chlorpyrifos & 1B \\
\hline M-Pede & potassium salts of fatty acids & no code \\
\hline Malathion & malathion & $1 \mathrm{~B}$ \\
\hline MBI-203 & Chromobacterium subtsugae & no code \\
\hline Mycotrol & Beauveria bassiana & no code \\
\hline Naturalis & Beauveria bassiana & no code \\
\hline Oberon & spiromesifen & 23 \\
\hline PFR-97 & Isaria fumosorosea & no code \\
\hline Platinum & acetamiprid & $4 \mathrm{~A}$ \\
\hline Portal & fenpyroximate & $21 \mathrm{~A}$ \\
\hline Preferal & Isaria fumosorosea & no code \\
\hline Provado & imidacloprid & $4 \mathrm{~A}$ \\
\hline PyGanic & pyrethrins & $3 \mathrm{~A}$ \\
\hline Pyrellin & pyrethrins \& rotenone & $3 A \& 21 B$ \\
\hline
\end{tabular}




\begin{tabular}{|c|c|c|}
\hline Trade Name Examples & Active Ingredient (common name) & Mode of Action Code \\
\hline Pyrenone & pyrethrins & $3 \mathrm{~A}$ \\
\hline Pyreth-It & pyrethrins & $3 A$ \\
\hline Radiant & spinetoram & 5 \\
\hline Rimon & novaluron & 15 \\
\hline Savey & hexythiazox & $10 \mathrm{~A}$ \\
\hline Sevin & carbaryl & $1 \mathrm{~A}$ \\
\hline Slug-Fest & metaldehyde & no code \\
\hline Sluggo & iron phosphate & no code \\
\hline SorbiShield & sorbitol octanoate & no code \\
\hline Spintor & spinosad & 5 \\
\hline SucraShield & sucrose octanoate & no code \\
\hline Synapse & flubendamide & 28 \\
\hline Telone & 1,3-dichloropropene & no code \\
\hline Trilogy & clarified hydrophobic extract of neem oil & no code \\
\hline Ultra-Fine Oil & refined petroleum distillate & no code \\
\hline Vapam & metam sodium & no code \\
\hline Vendex & fenbutatin oxide & $12 \mathrm{~B}$ \\
\hline Voliam Flexi & thiamethoxam \& chlorantraniliprole & $4 A \& 28$ \\
\hline Xentari & Bacillus thuringiensis aizawai & $11 \mathrm{~A}$ \\
\hline Zeal & etoxazole & $10 \mathrm{~B}$ \\
\hline \multicolumn{3}{|c|}{$\begin{array}{l}\text { a"un" means this compound has an unknown or uncertain mode of action. } \\
\text { bWhen no mode of action code is present, there is no code established and the product can be used without regard to mode of action. }\end{array}$} \\
\hline
\end{tabular}

